\title{
Research and Application of Coal Mine Safety Monitoring System Based on Communication Technology
}

\author{
Zhen Yang \\ Lanzhou Resource and Environment Voc-Tech College, Electrical and Mechanical Department, Lanzhou, \\ 730021, China
}

Keywords: Coal Mining, Communications Technology, Equipment Management.

\begin{abstract}
In the process of coal mine production, the dependence on working face equipment is very large, so the maintenance and management of working face equipment has a very important role, which can effectively improve the efficiency of coal mine production and avoid bad production accidents. With the deepening of the mining degree of mineral resources in China, the mineral industry itself has been developed and built for a long time, in which a lot of mining equipment has been used. In some mines with long mining time, we should improve the efficiency of mining and increase the strength and strength of mining. The research and application of coal mine safety monitoring system based on communication technology is a key link in coal mine production, which is of great practical significance and economic value.
\end{abstract}

\section{Introduction}

The use of equipment in coal mine production process is often in the high-load operation state, the working environment has its own particularity, therefore, it is also very common to have equipment problems in coal mine production, which will affect the economic benefits of enterprises, even threaten the personal safety of production workers, and hinder the safety of coal mine production work. In view of this, the safety management of coal mine production can effectively ensure the stable operation of production and promote the development and construction of related industries. The operation of coal mine safety monitoring and monitoring system can improve the stability and effectiveness of equipment use in the production process, reduce the hidden dangers and problems in the production process, and improve the efficiency in the process of coal mining.

\section{Supervision and Control of the Use of Equipment}

\subsection{Motor Use and Run}

In the current implementation of the field safety control motor in China, even in the overall has achieved corresponding results, but also to ensure the smooth use of many equipment. However, the equipment in the use of a large number of connection non-standard operation situation, resulting in overall construction quality safety problems, their own site safety control performance also needs to be considered.

The main reason is that the site safety control equipment needs to carry out complex and diverse wiring operations in the process of use, the staff's own professional ability is not very standard, and it is difficult to ensure that the equipment wiring process does not appear problems. In order to reduce the difficulty and strength of the work, some of the staff in the process of operation, in the process of wire erection blindly pursues the convenience and convenience, the line that should have been welded has not been welded in time, and the stability and fastness of the wiring of the equipment have been affected. And some of the staff cannot effectively understand the relevant standards and regulations of wiring, the way and means of wiring is not complete and unified, resulting in the phenomenon of random wiring, inducing certain safety problems and hidden dangers[1]. figure 1 for the four high voltage frequency converters used in coal mine production. 


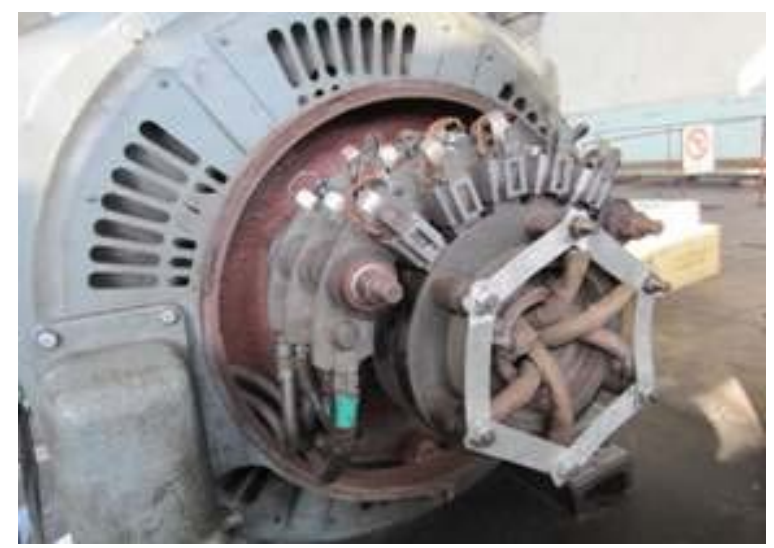

Figure 1 Four high voltage inverters

\subsection{Equipment Frequency Conversion and Speed Regulation}

In the process of reforming the motor, the winding and the stator winding should be rewired, and the copper wire with larger diameter should be selected. And in the process of the traditional motor work is basically the use of yellow wax cloth and other insulation materials, its own poor thermal conductivity, in the process of equipment work cannot effectively protect the temperature of equipment, improve the instability of equipment operation. After modification, polyimide film, polyamide fiber paper and polyester film can be used to protect the stability and safety of motor equipment. On the basis of ensuring the stable and efficient operation of the motor, the field operation control of the motor can be guaranteed. Only on the basis of the stable and scientific operation of the motor can a deeper field control be carried out to ensure that the motor can operate in a reasonable and effective direction.

\subsection{Improving Efficiency of Equipment}

In order to properly simplify the management system and process of the motor, a part of the automatic control system should be properly introduced, which can reduce the heavy work in the maintenance and management process, reduce the working pressure of the staff, and ensure that most of the equipment systems can run smoothly and effectively when the parts of some equipment are damaged. Furthermore, it can effectively improve the management and maintenance ability of the motor, and improve the power supply stability and safety of the motor. The choice of automatic control equipment can basically use the single-star Ethernet structure to design the computer network of the motor. It can effectively reduce the network communication effect of the motor and reduce the cost of modification to a certain extent[2].

The detailed frequency conversion research of the motor can reduce the energy consumption of the motor, and the energy consumption of the coal mine itself can save more than $70 \%$ of the power resources effectively compared with the unregulated equipment after the frequency conversion regulation, which improves the stability and effectiveness of the equipment operation, and ensures that the use of the coal mine equipment in the engineering construction can maintain more abundant economic benefits.

According to the above discussion, the coal mine in the operation of the lifting of the heavy weight of the goods, the electric power generated by the motor will be enhanced, the consumption of electricity will continue to increase. The main reason for a large amount of energy consumption in coal mines is that the rotor change in the motor cannot effectively affect the resistance of the motor, and the energy consumption of the reserve energy in the process of motor operation is large. The technology of "regenerative energy feedback device" should be introduced in the use of electric motor, which can effectively save electricity consumption and reduce the phenomenon of energy waste in coal mine use.

\subsection{Extension of Service Life of Equipment}

Through the discussion above, it can be proved that the important factor in the operation of coal 
mine is the power supply voltage regulation frequency modulation, which can effectively improve the renewable capacity in the use of power resources. Based on the analysis of the performance of the electric motor in the coal mine, reducing the resistance consumption in the operation of the equipment can greatly improve the economic benefit of the equipment. Introducing the technology of infinitesimal speed regulation can reduce the number of resistance in the use of equipment, and prolong the use effect and fluency of equipment.

According to the research of the current motor, it can be seen that the use of frequency converter to adjust the resistance mainly depends on the PLC controller to control the operation speed of the equipment, and the operation and AC of the electric power basically rely on the RS-485 interface end for frequency conversion use. Such a power operation system can effectively reduce the loss and consumption of power in the process of transmission, improve the equipment power system resource operation consumption phenomenon, enhance the pressure resistance and anti-attack ability of the equipment, the use of the lifting equipment for a long time.

\section{Application of Coal Mine Safety Monitoring and Monitoring System}

\subsection{Coal Mine Gas Monitoring Technology}

A large amount of gas is used in the process of coal mine production, in order to avoid the gas content in the coal mine exceeding the standard, the amount of ventilation needed in the production also has obvious requirements. In order to ensure that the fan can run well and stably, the double fan and double power supply can be used in the process of production, which can minimize the gas content in the coal mine and ensure the safety of the staff in production. However, in the process of practical application, we should minimize the problems and hidden dangers in the process of automatic switching of double fans, ensure the stable operation of the equipment, and then be able to effectively solve the use of communication technology in coal mine safety inspection. The automatic switching function of the local fan should include the following several different contents, first of all, it should realize the automatic fan switching, in the process of using a fan equipment problems, stop the work, should start the backup fan in time, the ventilation system in the coal mine environment to ensure smooth and stable. Secondly, the interval of the use of the fan should be guaranteed, and the other one should be in the rest stage during the operation of one typhoon machine, so as to avoid the phenomenon of air duct damage caused by the simultaneous operation of the two typhoon machines. Finally, when the two fans cannot work, it is necessary to ensure that the monitoring system can identify the operation of the equipment in a short time, and in the system terminal can timely issue the instructions to close the gas, to ensure the safety of coal mine operation. Figure 2 is a coal mine gas detector.

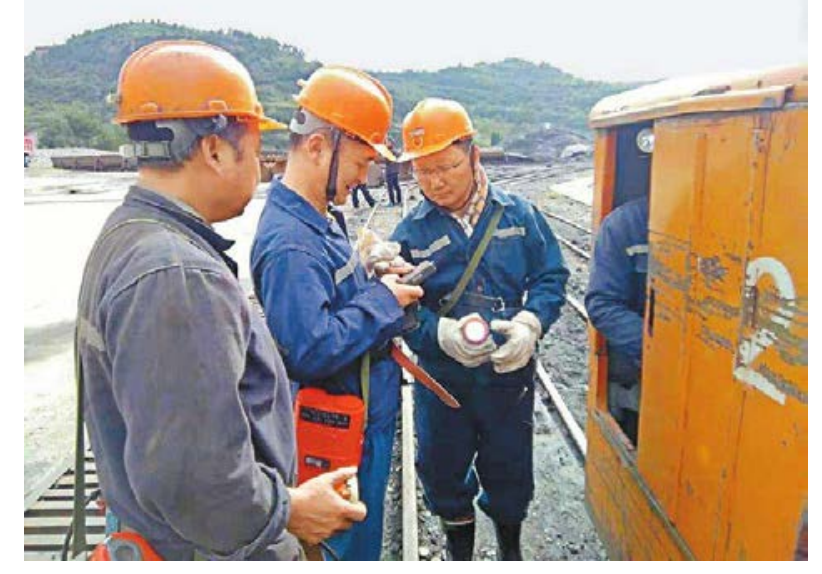

Figure 2 Coal mine gas testing

\subsection{Coal Mine Ventilation Safety Monitoring Technology}

The ventilation system should be included in the mine supervision and supervision under the guidance of the information system. The main work contents include control technology, electrical 
explosion protection, computer application, information transmission and so on. Specifically, it is necessary to monitor the environmental quality and various parameters in the mine in real time, supervise and monitor the temperature, humidity, carbon dioxide content and so on, adjust the working state of the equipment in the mine, and make a detailed analysis in the data integration to ensure the excellent working environment of the coal mine. as shown in figure 3 for coal mine ventilation equipment.

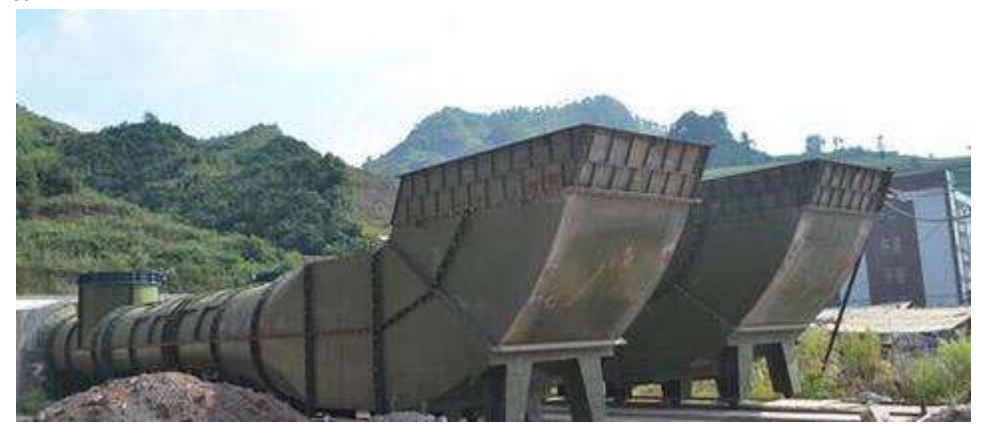

Figure 3 Coal mine vents

\section{Enhancing the Professional Competence of Operators}

Because coal mining in the present coal mining, social logistics and other aspects of extremely extensive use of efficiency, and then need a large number of professional and professional operators to control, reduce the use of equipment due to non-standard operation caused by equipment wastage and waste of resources. The effective training and guidance for the operators of coal mines can improve the failure and problems in the use of equipment and improve the efficiency of equipment use. The scientific and reasonable operation technology can also give the equipment more long-term effect, and the normal use form can improve the use time of the equipment and reduce the unnecessary loss[3].

\section{Conclusion}

According to the above discussion, the working face equipment plays an important role in coal mine production, and has an important influence on the quality and efficiency of coal mine production. Therefore, it is necessary to constantly strengthen the maintenance and nursing work of the working face equipment in the coal mine production, to ensure that the working face equipment can play its own functions in a relatively stable environment, to help the high efficiency and convenience of coal mine production, and to improve the stability and safety of coal mine production. Green coal mining processing cost control plays a very important role in China's coal mining industry, which can enhance the economic benefits within our industry and lay a solid foundation for the development of our industry.

\section{Acknowledgements}

Research project of Gansu Higher Education Institute, project number: 2018C-25.

\section{References}

[1] Tang, Zhigang. Discuss the application of monitoring technology in coal mine safety production. Construction Engineering Technology and Design, no. 29, pp. 3496, 2019.

[2] Cui, Xiuhua., Zhang, Jinhua. Analysis of coal mine safety monitoring and monitoring system. Inner Mongolia Coal Economy, no. 15, pp. 154-155, 2019.

[3] Mao, Kaijiang. Research and design of coal mine safety monitoring and monitoring data network acquisition system. China Coal, vol. 45, no. 11, pp. 49-52, 2019. 